\title{
Experimental and theoretical investigations of absorption and emission spectra of the light-emitting polymer MEH-PPV in solution
}

\author{
R. Chang ${ }^{\text {a, * }}$, J.H. Hsu ${ }^{\text {a,b }}$, W.S. Fann ${ }^{a, b}$, K.K. Liang ${ }^{c}$, C.H. Chang ${ }^{c}$, M. Hayashi ${ }^{a}$, \\ J. Yu ${ }^{\text {d }, ~ S . H . ~ L i n ~}{ }^{\text {a }}$, E.C. Chang ${ }^{\text {e }}$, K.R. Chuang ${ }^{\text {e }}$, S.A. Chen ${ }^{\mathrm{e}}$ \\ ${ }^{a}$ Institute of Atomic and Molecular Sciences, Academia Sinica, P.O. Box 23-166, Taipei, 106 Taiwan \\ ${ }^{\mathrm{b}}$ Department of Physics, National Taiwan University, Taipei, Taiwan \\ ${ }^{c}$ Department of Chemistry, National Taiwan University, Taipei, Taiwan \\ ${ }^{\mathrm{d}}$ Department of Chemistry, National Kaoshiung Normal University, Kaoshiung, Taiwan \\ e Department of Chemical Engineering, National Tsing-Hwa University, Hsinchu, Taiwan
}

Received 5 October 1998; in final form 6 August 1999

\begin{abstract}
We report the absorption and photoluminescence spectra of the MEH-PPV polymer in a chloroform solvent. In the analysis, we assume that the polymer consists of short conjugated segments which are called oligomers with different lengths. The appearance of each chain length is controlled by a distribution function. Based on the conformational disorder caused by torsional motion, we found that the distribution function should take a Gaussian form with its center and width being adjustable parameters. While the energy level of the emissive $S_{1}$ state of the oligomer and the corresponding oscillator strength are calculated from exciton theory. In addition, the associated vibrational motions are considered in determining the spectral shape in which both displacement and distortion of the potential surfaces are taken into account. With this information, the calculation result can fit the experiment reasonably well. (c) 2000 Elsevier Science B.V. All rights reserved.
\end{abstract}

\section{Introduction}

In the last decade, PPV and its derivatives have been extensively studied in a variety of experiments using molecular spectroscopy. Absorption, photoluminescence (PL) and photoluminescence excitation (PLE) have been applied to determine the electronic states and optical properties [1-4]. Infrared, Raman and resonant Raman techniques have been used to analyze the ground-state vibrational modes of PPV [2,4-6], and detailed structural information has been obtained. Moreover, the dynamics of excited species has been investigated by time-resolved PL [7,8] and photoinduced absorption (PA) methods [9,10].

However, in theoretical investigations, two conflicting arguments - one-dimensional semiconductor band theory and molecular exciton theory - have been proposed. The former is mostly based on the Su-Schrieffer-

\footnotetext{
* Corresponding author: Fax: + 886-2-3620200; e-mail: richang@po.iams.sinica.edu.tw
} 
Heeger (SSH) model [11] in which the electron-electron correlation and Coulomb interaction are totally ignored [12] and strong electron-phonon coupling is assumed. Additionally, the PL is generated as a consequence of recombination of the free electron-hole pair whereas, in the latter model, the emissive excited states are assumed to be singlet excitons localized in a finite chain.

Although the band theory has been successful in explaining a number of experiments [13-15], there is some reason to justify the use of molecular exciton theory. It is generally accepted that these phenylene-based conjugated systems acquire a planar configuration. However, the randomly appearing torsion angle between adjacent monomer units effectively interferes with the behavior of the $\pi$-electron so that the conjugation length is much shorter than the physical length of the whole polymer. Therefore, it gives rise to statistical variation in conjugation lengths and in the results for the broad band absorption spectrum [16-20].

Conceptually, there are two different mechanisms related to a torsion angle that lead to a finite $\pi$-conjugation length in polymer, namely abrupt flips [21,22] and conformational disorder [23]. The former considers that the almost planar conjugated segments are separated by the relatively large torsion angles. However, in the latter mechanism, the wavefunction of the $\pi$-electron cannot extend to infinite as a result of interference due to the disordered arrangement of torsion angles. In a one-dimensional disorder system, it is well known that, no matter how weak the disorder, the electron is localized to some extent [24]. The finite conjugation length caused by abrupt flips is a concept of classical limit; once the torsion angle is greater than some critical value, forming the flip (break), the electrons on both sides of the flip cannot correlate each other (i.e. quantum tunneling is not effective). The critical value of the torsion angle plays a key role in obtaining the distribution. Previous works based on the abrupt flips model, e.g., Kohler et al. [21] or Yaliraki et al. [22], have chosen a relatively small critical value so that the distribution is peaked at the shortest length and the decay with chain length behaves exponentially. Once the critical angle chosen is increased to a certain value, the infinite long chain (i.e. equal to the length of the whole polymer) dominates the distribution. However, the existence of perfect conjugation can be hampered by the conformational disorder, i.e. the second mechanism explained above.

In previous experimental works, Shand et al. [25] studied polyacetylene in solution, and resonance Raman as well as absorption spectra were taken. In their analysis, a modified Gaussian function is used to model the conjugation length distribution. The absorption profile was assumed to be Lorentzian while the center frequency was a decreasing function of the conjugation length. Kohler et al. [21] investigated the same system and calculated the transition dipole moments and frequencies based on the Hückel model, and the absorption profile was obtained from $\beta$-carotene. The distribution function peaks at the shortest segment when the total chain length is very long. Meanwhile the width of the distribution increases with the total chain length.

In this Letter, we study the dilute MEH-PPV solution in chloroform and show that the distribution of the chain length is more likely to be determined by conformational disorder in our experiment in contrast to the work in Refs. [21,22]. This is consistent with the result of the quantum chemistry calculation [26] which shows that MEH-PPV has a relatively small range of torsion angle at room temperature. As a result, the distribution function takes a Gaussian form. We determine the center and width of the Gaussian distribution so that the absorption and PL spectra evaluated by superpositions of the profiles for the oligomer of PPV with different chain lengths can fit the experiment. The transition moments and transition frequencies of the oligomer are obtained from an exciton model. The profiles are dominated by the vibronic coupling constants of the high-frequency modes. The coupling constant, transition frequency and transition moment are all determined by the chain length of the oligomer.

\section{Experimental}

MEH-PPV is prepared following a procedure similar to that used by Wudl et al. [27]. The polymers are dissolved in the chloroform solution. The absorption spectra are recorded on a Hitachi U-3200 spectrophotom- 


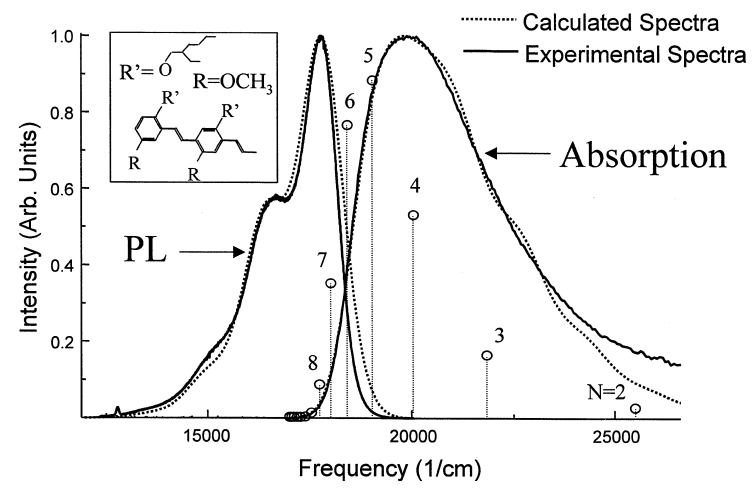

Fig. 1. Experimental and calculated cw photoluminescence and absorption for MEH-PPV in chloroform solution. Circles indicate the total absorption coefficients $D[N]\left|\vec{\mu}_{N}^{1}\right|^{2}$ of N-unit oligomer in the abscence of vibrations. Inset: the molecular structure of MEH-PPV.

eter under ambient conditions. The fluorescence spectra are recorded by using a liquid nitrogen-cooled CCD-based system and excited at $420 \mathrm{~nm}$. The liquid samples are under nitrogen bubbling before experimentation.

The observed PL as well as absorption spectra are displayed in Fig. 1 together with the results from calculation. The width of the PL spectrum is about one-half of the absorption and the PL spectrum has a vibronic feature in the shoulder.

\section{Theory}

In this section, we will briefly present the basic theoretical approach used in the analysis of spectra. In general, with vibrational motion taken into account, the absorption coefficient for the electronic transition $a \rightarrow b$ can be expressed as [28,29]

$$
\alpha_{a b}(\omega)=\frac{2 \pi \omega}{3 a c \hbar}\left|\vec{\mu}_{b a}\right|^{2} \int_{-\infty}^{\infty} \mathrm{d} t \exp \left[\frac{\mathrm{i} t}{\hbar}\left(E_{b}-E_{a}-\hbar \omega\right)-d^{2} t^{2} / 2\right] \prod_{j} G_{j}(t)
$$

where $\vec{\mu}_{b a}$ denotes the electronic transition moment, $a$ the factor which describes the medium effect, $c$ the speed of light, $d$ the width of inhomogeneity of electronic states and

$$
\begin{aligned}
G_{j}(t)= & \frac{2 \beta_{j} \beta_{j}^{\prime \prime} \sinh \left(\hbar \omega_{j} / 2 k T\right)}{\sinh \left(\lambda_{j}\right) \sinh \left(\mu_{j}^{\prime \prime}\right)} \\
& \times \frac{\exp \left[-\beta_{j}^{2} \beta_{j}^{\prime \prime 2} \Delta Q_{j}^{\prime \prime 2} /\left(\beta_{j}^{\prime \prime 2} \operatorname{coth}\left(\lambda_{j} / 2\right)+\beta_{j}^{2} \operatorname{coth}\left(\mu_{j}^{\prime \prime} / 2\right)\right)\right]}{\left[\left(\beta_{j}^{\prime \prime 2} \operatorname{coth}\left(\mu_{j}^{\prime \prime} / 2\right)+\beta_{j}^{2} \operatorname{coth}\left(\lambda_{j} / 2\right)\right)\left(\beta_{j}^{\prime \prime 2} \tanh \left(\mu_{j}^{\prime \prime} / 2\right)+\beta_{j}^{2} \tanh \left(\lambda_{j} / 2\right)\right)\right]^{1 / 2}} .
\end{aligned}
$$

In Eq. (2),

$$
\begin{aligned}
& \beta_{j}=\left(\frac{\omega_{j}}{\hbar}\right)^{1 / 2}, \quad \Delta Q_{j}^{\prime \prime}=Q_{j}^{\prime \prime}-Q_{j}, \\
& \lambda_{j}=\mathrm{i} t \omega_{j}+\frac{\hbar \omega_{j}}{2 k T}, \quad \mu_{j}^{\prime \prime}=-\mathrm{i} t \omega_{j}^{\prime \prime},
\end{aligned}
$$


where $\omega_{j}$ and $\omega_{j}^{\prime \prime}$ are the oscillator frequencies of the $j$ th mode in the electronic states $a$ and $b$, respectively, $\Delta Q_{j}^{\prime \prime}$ denotes the normal coordinate displacement. For emission spectra, one can obtain a similar expression given by

$$
I_{b a}(\omega)=\frac{2 \pi a \omega^{3}}{3 c \hbar}\left|\vec{\mu}_{b a}\right|^{2} \int_{-\infty}^{\infty} \mathrm{d} t \exp \left[\frac{i t}{\hbar}\left(E_{b}-E_{a}-\hbar \omega\right)-d^{2} t^{2} / 2\right] \prod_{j} \bar{G}_{j}(t)
$$

where $\bar{G}_{j}(t)=G_{j}^{*}(t)$ with $\omega_{j}$ and $\omega_{j}^{\prime \prime}$ exchanged. For the case of pure displacement, i.e. $\omega_{j}=\omega_{j}^{\prime \prime}$ and $\Delta Q_{j}^{\prime \prime} \neq 0$. We will include the distortion effect $\omega_{j} \neq \omega_{j}^{\prime \prime}$ since the asymmetry has been observed in the short oligomer [32].

We will consider a situation in which there is more than one species, i.e. a distribution of conjugation lengths. For that, the absorption coefficient is given by

$$
\alpha(\omega)=\sum_{N} D[N] \alpha_{b_{N} a_{N}}(\omega)
$$

where $D[N]$ denotes the distribution of the $N$-unit oligomer in the sample, and PL spectrum is

$$
I(\omega)=\sum_{N} D[N] \rho_{b_{N}} I_{b_{N} a_{N}}(\omega)
$$

where $\rho_{b_{N}}=\exp \left(-E_{b_{N}} / k_{\mathrm{B}} T\right) / \sum_{N} \exp \left(-E_{b_{N}} / k_{\mathrm{B}} T\right)$. In Eq. (6), we have assumed that the thermal equilibrium within all electronic states has been reached prior to the emission.

\section{Results and discussions}

\subsection{Oligomer properties}

As mentioned in the previous section, the conjugation length of polymers in solution is a broad distribution. We will simulate the spectra on the basis of oligomer properties. The structure of the oligomer used to model the polymer system is shown in Fig. 1 for two units as an example. In the exciton theory, a conjugated system of linear geometry and with $N$ units can have energies

$$
E_{l}=E_{0}+2 \beta \cos \left(\frac{\pi l}{N+1}\right)
$$

where $l=1,2, \ldots N, E_{0}$ is the energy of excited state of each unit and $\beta$ is the interaction strength between nearest-neighbor units. The corresponding wavefunction is

$$
\Phi_{l}=\sum_{n=1}^{N} a_{n}^{l} \psi_{n}
$$

and the transition dipole moment is

$$
\vec{\mu}_{N}^{l}=\sum_{n=1}^{N} a_{n}^{l} \vec{\mu}_{n}
$$

with

$$
a_{n}^{l}=\sqrt{\frac{2}{N+1}} \sin \left(\frac{\pi n l}{N+1}\right),
$$


$\vec{\mu}_{n}$ being the transition moment of each unit. When $\vec{\mu}_{n}=\vec{\mu}$ for all units, we find

$$
\left|\vec{\mu}_{N}^{l}\right|^{2}=\frac{2|\vec{\mu}|^{2}}{N+1}\left[\cot \left(\frac{\pi l}{2 N+2}\right)\right]^{2}
$$

for $l=1,3, \ldots 2 n+1 \ldots$ Obviously the transition dipole moment for exciton state is a decreasing function of $l$. Previous work performed by $\mathrm{Yu}$ et al. [18] gives $E_{0}=5.0 \mathrm{eV}\left(40000 \mathrm{~cm}^{-1}\right)$ and $\beta=-1.1 \mathrm{eV}\left(8800 \mathrm{~cm}^{-1}\right)$ by fitting the value of $E_{0}$ and $\beta$ to the molecular orbital calculations for PPV oligomer. In this case, the state $\mathrm{S}_{1}(l=1)$ has the largest transition moment but lowest energy. Therefore $S_{1}$ is the primary state that contributes to the observed $\mathrm{cw}$ luminescence. The energy of $\mathrm{S}_{1}$ state decreases with the chain length $N$. In applying Eq. (7) to fit our spectra, we choose the same $\beta$ while $E_{0}$ becomes a fitting parameter. As a result of the alkoxy side chain in each unit, $E_{0}$ must be significantly smaller than pure PPV. We choose $E_{0}=4.3 \mathrm{eV}\left(34400 \mathrm{~cm}^{-1}\right)$ so that the calculated absorption spectrum is red-shifted so that further adjustment of other parameters including the chain length distribution can steadily fit the spectra. The $N$-dependent behavior of energy levels $E_{1}$ and the transition moment $\left|\vec{\mu}_{N}^{1}\right|$ is illustrated in Fig. 2 where the monomer dipole moment $|\vec{\mu}|^{2}=1$. The energy level is sensitively dependent on chain length in particular at the short region (e.g., $N<5$ ).

We now examine the vibrational contribution to the spectra of oligomers. According to a previous study of oligomer MPV, a derivative of PPV, we found that the high-frequency vibrational mode in excited state has higher frequencies than those in the ground state [32]. Thus both displacement and distortion have to be considered in calculating the spectra. For the oligomers considered here, we will also take into account displacement as well as distortion. The vibrational modes chosen are 1400 (1550), 700 (700) and 200 (200) $\mathrm{cm}^{-1}$ for ground (excited) state for all the oligomers. The existence of modes 1400 and $700 \mathrm{~cm}^{-1}$ have been justified in previous Raman as well as IR spectra [5,6]. The corresponding displacement is chosen so that $S_{i} \equiv \omega_{i} \Delta Q_{i}^{2} / 2 \hbar=a_{i}+b_{i} /(N+1)$ with $a_{i}$ and $b_{i}$ being adjustable. The $N$-dependent $S_{i}$ is consistent with the previous study of oligomer PL spectra which show that the coupling constant is a decreasing function of chain length $N$ [20,30]. In Appendix A, we give a derivation to show the Huang-Rhys factor as an increasing function of $1 /(N+1)$ in order to support this fact qualitatively. We choose $a_{1400}=0.15, b_{1400}=2.5$, $a_{700}=0.006, b_{700}=0.153, a_{200}=0.1$, and $b_{200}=18.0$, i.e. $S_{1400}\left(S_{700}, S_{200}\right)=0.57(0.026,3.1)$ for $N=5$. The large $S$ value for the $200 \mathrm{~cm}^{-1}$ mode is essential in order to fit the large Stokes shift and can be regarded as a result due to the existence of many modes in the low-frequency region. In calculating the spectra, we fix the temperature at $T=300^{\circ} \mathrm{K}$. Therefore, the absolute value of the low-frequency mode is not so sensitive but has to be chosen so that $\sum_{i} S_{i} \omega_{i}$ which controls the magnitude of Stokes shift is with a fixed value. In a previous study of MPV oligomer whose backbone structure contains three-unit benzene rings, the Stokes shift has been about $1000 \mathrm{~cm}^{-1}$, and we used $S_{200}=5.0$ for fitting; here we use $S_{200}=4.5$ for $N=3$.

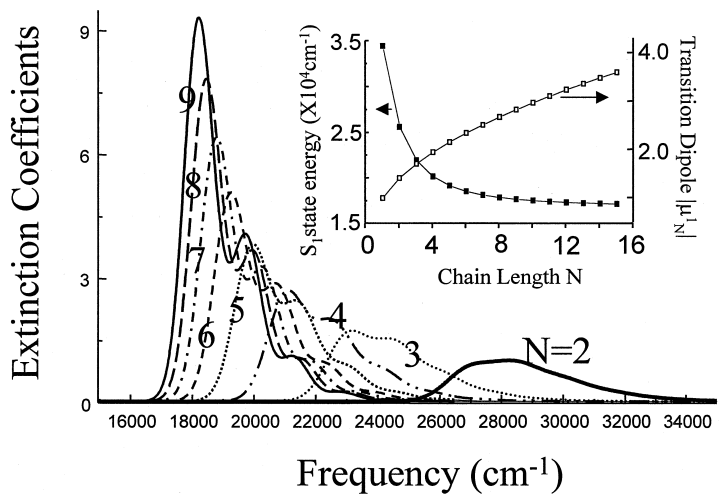

Fig. 2. Calculated absorption spectra in extinction coefficient for oligomer with $N=2-9$. All the profiles are normalized to the peak of $N=2$. The inset shows the $\mathrm{S}_{1}$ state energy and transition dipole magnitude for different chain lengths $N$. 


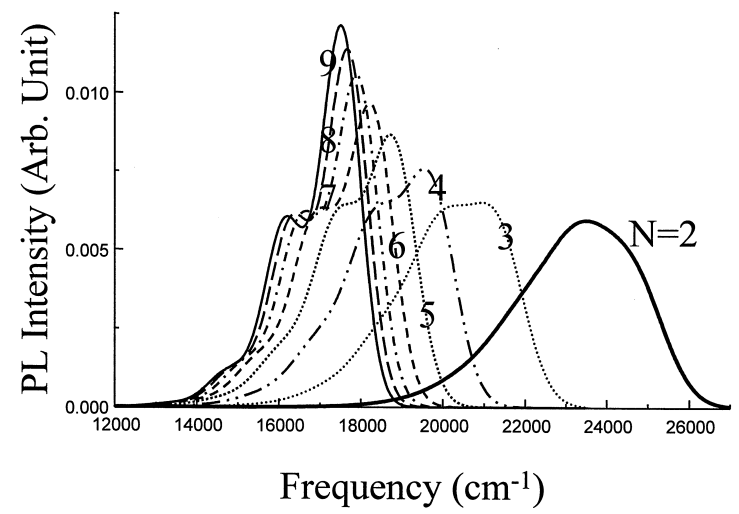

Fig. 3. Calculated PL spectra for oligomer with $N=2-9$. All profiles have been normalized to unity area.

The absorption and PL spectra for each oligomer are shown in Figs. 2 and 3, respectively. Fig. 3 is normalized so that all the PL profiles have an area equal to unity. In Fig. 2, the absorption profiles are displayed in extinction coefficients normalized to the peak of $N=2$.

\subsection{Chain length distribution}

Prior to the calculation of absorption and emission spectra, we have to treat the key issue of distribution of conjugation length. As explained in Section 1, the abrupt flips and conformational disorder are the two main causes that account for the finite chain length. Considering an $M$-unit polymer which have $M-1$ independent torsion angles, we can write the effective potential for them as $V=\sum_{i=1}^{M-1} V\left(\phi_{i}\right)$ with $\phi_{i}$ being the torsion angle between $i$ and $i+1$ units. In the following discussion, a simple form $V\left(\phi_{i}\right)=(1 / 2) K \phi_{i}^{2}$ is assumed that has a minimum when all units are aligned. We first present some results of the abrupt flips model by numerical simulation. In a state of thermal equilibrium, the distribution of each torsion angle takes the form

$$
P(\phi)=\frac{\exp [-\beta V(\phi)]}{\int \exp [-\beta V(\phi)] \mathrm{d} \phi}
$$

where $\beta=1 / k_{\mathrm{B}} T$. In an abrupt flips model, as $\phi>\phi_{c r}$, the conjugation is broken and we can evaluate $D[N]$, the distribution of length using the Metroplis method [31], the same one used by Yaliraki et al. [22]. Results of different $\phi_{c r}$ are demonstrated in Fig. 4 for a polymer with a total number of units $M=40$ and $K=3000$ $\mathrm{cm}^{-1}$. In Fig. $4 \mathrm{a}, \phi_{c r}=0.2$, the distribution function takes the exponentially decaying form peaked at $N=1$. This is a behavior similar to that obtained by Yaliraki et al. [22]. With the increase of $\phi_{c r}$, we observe a large increase in the longest chain as shown in Fig. 4b,c where the values $\phi_{c r}=0.55$ and 0.7 are chosen respectively. Fig. 4c(4a) corresponds to a polymer having conjugation that can (not) sustain larger torsion angle. An accurate determination of $\phi_{c r}$ is not a straightforward task, but through an empirical approach we rule out the possibility of a small $\phi_{c r}$. We have attempted to fit our result by using an exponential distribution $D[N]=\exp (-\lambda N)$ corresponding to the feature of Fig. 4a but the fitting is not successful. The reason is briefly outlined in Fig. 5 . where we plot the total absorption coefficients without vibrational part, i.e. $D[N]\left|\vec{\mu}_{N}^{1}\right|^{2}$ versus energy with $\vec{\mu}_{N}^{1}$ and energy being obtained from Eqs. (9) and (7), respectively. The one with $\lambda=0.05$ (hollow circle) is so chosen in order to have the width of absorption broader than that of emission by about $2000 \mathrm{~cm}^{-1}$ (as observed in experiment shown Fig. 1), but it peaks at $N=15$ that will have an emission spectrum too smooth to have vibronic feature according to above explanation of chain length dependence of coupling constants. Meanwhile the resulting Stokes shift must be small unless a large inhomogeneity $\sim 1000 \mathrm{~cm}^{-1}$ is chosen, which will also 


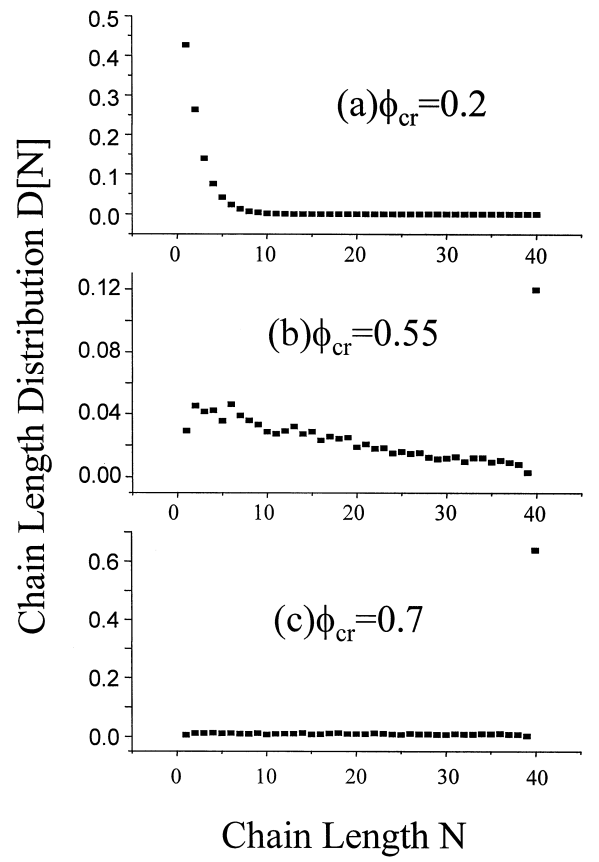

Fig. 4. The chain length distribution calculated using the Metroplis method for total length $M=40$ using the abrupt flips model. The critical angle $\phi_{c r}$ is (a) 0.2 , (b) 0.55 , (c) 0.7 .

lead to a structureless emission profile. While the choice of $\lambda=0.2$ (diamond cross) can lead to a reasonable Stokes shift in the fitting. In addition, since it peaks at $N=6$, the emission can be fitted reasonably. Nevertheless the width is much broader than the observed one, it will be even more broadened once vibrational part is included. Therefore we conclude that abrupt flips model is not a likely mechanism in describing conjugation length distribution as far as our system is concerned.

In the following, we will take for granted that $\phi_{c r}$ is large enough in our system that the conjugation length defined by abrupt flips model is almost the same as the whole polymer. While we will investigate if the

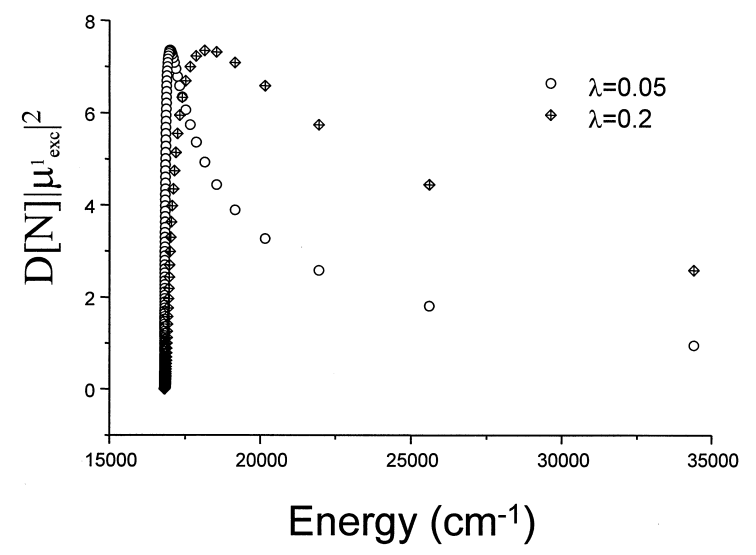

Fig. 5. Total absorption coefficients $D[N]\left|\vec{\mu}_{N}^{1}\right|^{2}$ of $N$-unit oligomer, with distribution $D[N]=\exp (-\lambda N)$, a typical result obtained for small critical angle in abrupt flips model for $\lambda=0.05$ (hollow circles) and $\lambda=0.2$ (diamond cross). 
alternative definition of conjugation based on conformational disorder model will lead to a proper distribution of length for fitting. In order to describe conformational disorder, Rossi et al. [23] defined a correlation function

$$
P_{L}=\cos ^{2} \theta_{0 L}-\frac{1}{2}
$$

where $\theta_{0 L}$ is the relative angle between normal vectors on the 0 th and $L$ th aromatic units. They have proved that the ensemble average of Eq. (12) obtained by the partition function $Z=\prod_{i=1}^{L}\left\{\int \exp \left[-V\left(\phi_{i}\right) / k_{\mathrm{B}} T\right] \mathrm{d} \phi_{i}\right\}$ leads to

$$
\left\langle P_{L}\right\rangle=\frac{1}{2}\langle\cos 2 \phi\rangle^{L} \equiv \frac{1}{2} \exp \left(-\frac{L}{\lambda_{c}}\right) .
$$

The correlation lengths $\lambda_{c}=1 /|\ln (\eta)|$ is defined by Rossi et al. [23]. as the effective conjugation length though they are descriptions of conformational structure. One can easily prove that for $1-\langle\cos 2 \phi\rangle \ll 1$,

$$
\lambda_{c} \approx 2 \sum_{j=i+1}^{\infty}\left\langle P_{j-i}\right\rangle \equiv\left\langle\lambda^{\prime}\right\rangle
$$

where $\lambda^{\prime}$ is defined in a form that allows us to evaluate the distribution function of conjugation length. Notice that an equation similar to Eq. (14) was derived by Rossi et al. [23] in treating the persistence length. According to the central limit theorem, the distribution of $\lambda^{\prime}$ is definitely in Gaussian form with a width $\sigma_{c}$ satisfying

$$
\sigma_{c}^{2}=4 \sum_{j=i+1}^{\infty}\left\langle\left(P_{j-i}-\left\langle P_{j-i}\right\rangle\right)^{2}\right\rangle .
$$

Moreover, one can prove by simple algebra that $\left\langle\left(P_{j-i}-\left\langle P_{j-i}\right\rangle\right)^{2}\right\rangle=\Delta C^{2}\left\langle\left(P_{j-i-1}-\left\langle P_{j-i-1}\right\rangle\right)^{2}\right\rangle$ with $\Delta C^{2} \equiv\left\langle(\cos 2 \phi-\langle\cos 2 \phi\rangle)^{2}\right\rangle$. By using Eq. (13), we obtain

$$
\sigma_{c}^{2}=\frac{\Delta C^{2}}{1-\Delta C^{2}} .
$$

We need empirical value to calculate $\Delta C^{2}$. Previously Lhost and Bredas [26] performed an ab initio calculation for 1,1'-(trans-1,2-ethene-diyl) bis-2,5-methoxybenzene using Gaussian 3-21G level. This compound possesses almost the same structure as the backbone of MEH-PPV and they found that it has quite rigid planarity in room temperature due to the formation of hydrogen bonding between methoxy group and the hydrogen on vinylene group. According to their result in room temperature $\Delta \phi \approx 0.383\left(22^{0}\right)$, namely $K \sim 14 k_{\mathrm{B}} T$. Since $V(\phi)=$ $(1 / 2) K \phi^{2}$, we have $\langle\cos 2 \phi\rangle \approx 1-2 k_{\mathrm{B}} T / K$ and $\Delta C^{2} \approx 3 k_{\mathrm{B}} T / K$. Then it follows that $\lambda_{c} \sim 7$, and $\sigma_{c} \sim 0.6$. The fitting using such estimated values of parameters is not successful because $\sigma_{c}$ is too small. An alternative correlation function defined as $P_{L}=\cos \theta_{0 L}$ [23,33] leading to a divergent $\sigma_{c}$ cannot fit either. Therefore a more sophisticated definition of correlation function for conformational disorder has to be defined in order to properly describe the corresponding conjugation of $\pi$-electron. However we will use an empirical way to calculate the spectra. Taking for granted the Gaussian distribution deduced from conformational disorder model, i.e.

$$
D[N]=\frac{1}{\sqrt{B_{\mathrm{av}}}} \exp \left[-\left(N-N_{0}\right)^{2} / B_{\mathrm{av}}\right] .
$$

where both center $N_{0}$ and width $\sqrt{B_{\text {av }}}$ are adjustable parameters, we evaluate spectra by Eqs. (5) and (6). With the spectral information about oligomers outlined in Figs. 2 and 3 , the best fit gives us $N_{0}=5$ and $\sqrt{B_{\mathrm{av}}}=1.8$. One point that might be noteworthy is our fitting result $N_{0}=5$ is not qualitatively different from the estimate $\left(\lambda_{c} \sim 7\right)$ mentioned above. The result of fitting is shown in Fig. 1 to compare with experiment. The emission and the red portion of absorption is quite reasonably fitted. Fig. 1 also illustrates the values of $D[N]\left|\vec{\mu}_{N}^{1}\right|^{2}$ by 
hollow circles and one can recognize it has caught the feature of absorption even before the vibrational part is taken into account. The fact that Gaussian distribution can fit experiment better than exponential distribution suggests that conformational disorder model is a more appropriate mechanism to describe the conjugation of MEH-PPV.

The deviation between the calculation and the experimental result in the blue tail could come from two effects. First, the Gaussian distribution might have underestimated the contribution from the short conjugation length that is the abrupt flips model still somehow take effect. The second is that there are actually higher excited states for each finite oligomer, and the finite value at the high frequency region could be resulted from the red tail of those states [34].

\section{Conclusions}

In conclusion, we have calculated the absorption and PL spectra of MEH-PPV polymer in solution. The polymer is modeled as a distribution of oligomers with varied conjugation lengths. The electronic state and the associated oscillator strength are obtained by the exciton theory. While in calculating the absorption and PL spectra for the oligomers, the displacement and distortion of the vibrational mode are taken into account. As for the distribution function, we have compared the features due to abrupt flips and conformational disorder, the two commonly accepted models. We found that MEH-PPV is in the region where the conformational disorder model can describe better and give a Gaussian distribution. Since we have not obtained the exact distribution profiles without any adjustable parameter, our understanding about these two models is far from complete. However the current empirical treatment has made us believe that the structure of MEH-PPV is rigid that torsional motion cannot break the co-planity effectively in room temperature. It will be an interesting work to systematically compare these two models on various other conjugated polymers.

\section{Acknowledgements}

This work was supported by Academia Sinica and the NSC of the Republic of China (NSC 87-2216-E-001002).

\section{Appendix A}

In this appendix, we shall show that the Huang-Rhys factor decreases with the chain length. Consider a polymer with $N$ units, each of which has one excited electronic state with identical energy and transition dipole moment. Then the exciton state with largest transition moment is given by

$$
\Phi_{1}=\sum_{n=1}^{N} a_{n} \psi_{n}
$$

where

$$
a_{n}=\sqrt{\frac{2}{N+1}} \sin \left(\frac{\pi n}{N+1}\right) .
$$

The energy of this state is

$$
E_{1}=E_{0}+2 \beta \cos \left(\frac{\pi}{N+1}\right)
$$


where $E_{0}$ is the excited state energy of each unit and $\beta$ is the interaction strength between nearby neighboring units. Then the total potential energy of the system is

$$
U=\left\langle\Phi_{1}\left|\sum_{n=1}^{N} U_{n}\right| \psi_{n}\right\rangle\left\langle\psi_{n} \mid \Phi_{1}\right\rangle
$$

where $\psi_{n}$ is the state in which $n$th unit is excited and $U_{n}$ is the corresponding potential energy. We assume that there is only one mode in each unit, then

$$
U_{n}=\frac{1}{2} \omega_{n}^{2}\left(Q_{n}+d_{n}\right)^{2}+\frac{1}{2} \sum_{n^{\prime} \neq n}^{N} \omega_{n^{\prime}}^{2} Q_{n^{\prime}}^{2}
$$

where $d_{n}$ is the displacement of potential surface. By inserting Eq. (A.1) into Eq. (A.4), we obtain

$$
\begin{aligned}
& U=\frac{1}{2} \sum_{n=1}^{N} \omega_{n}^{2} Q_{n}^{2}+\frac{2}{N+1} \sum_{n=1}^{N} \sin ^{2}\left(\frac{\pi n}{N+1}\right) \omega_{n}^{2} Q_{n} d_{n}+\ldots \\
& U=\frac{1}{2} \sum_{n=1}^{N} \omega_{n}^{2}\left(Q_{n}+d_{n}^{\prime}\right)^{2}+\ldots
\end{aligned}
$$

where

$$
d_{n}^{\prime}=\frac{2}{N+1} \sin ^{2}\left(\frac{\pi n}{N+1}\right) d_{n} .
$$

In case where vibration frequency $\omega_{n}$ and displacement $d_{n}$ on each site are identical, the total Huang-Rhys factor is

$$
\begin{aligned}
S & =\sum_{n=1}^{N} \frac{1}{2} \omega_{n} d_{n}^{\prime 2} \\
& =\frac{3}{2(N+1)}\left(\frac{1}{2} \omega d^{2}\right)=\frac{3}{2(N+1)} S_{\text {mono }}, \text { for } N>1
\end{aligned}
$$

which decreases with the chain length $N$.

\section{References}

[1] K. Pichler, D.A. Halliday, D.D.C. Bradley, P.L. Burn, R.H. Friend, A.B. Holmes, J. Phys.: Condens. Matter 5 (1993) 7155.

[2] S.C. Graham, D.D.C. Bradley, R.H. Friend, C.W. Spangler, Synth. Met. 41/43 (1991) 1277.

[3] T.W. Hagler, K. Pakbaz, A.J. Heeger, Phys. Rev. B 44 (1991) 8652.

[4] S. Heun, R.F. Mahrt, A. Greiner, U. Lemmer, H. Bassler, D.A. Halliday, D.D.C. Bradley, P.L. Burn, A.B. Holmes, J. Phys.: Condens. Matter 5 (1993) 247.

[5] B. Tian, G. Zerbi, K. Muellen, J. Chem. Phys. 95 (1991) 3198.

[6] B. Tian, G. Zerbi, K. Muellen, J. Chem. Phys. 95 (1991) 3191.

[7] R. Kersting, U. Lemmer, R.F. Mahrt, K. Leo, H. Kurz, H. Bassler, E.O. Gobel, Phys. Rev. Lett. 70 (1993) 3820.

[8] G.R. Hayes, I.D.W. Samuel, R.T. Phillips, Phys. Rev. B 52 (1995) 11569.

[9] J.W.P. Hsu, M. Yan, T.M. Jedju, L.J. Rothberg, Phys. Rev. B 49 (1994) 712.

[10] J.M. Leng, S. Jeglinski, X. Wei, R.E. Benner, Z.V. Vardeny, F. Guo, S. Masumdar, Phys. Rev. Lett. 72 (1994) 156.

[11] W.P. Su, J.R. Schrieffer, A.J. Heeger, Phys. Rev. Lett. 42 (1979) 1698.

[12] A.J. Heeger, S. Kivelson, R.J. Schrieffer, W.P. Su, Rev. Mod. Phys. 60 (1988) 782.

[13] R.H. Friend, D.D.C. Bradley, P.D. Townsend, J. Phys. D 20 (1987) 1367.

[14] T.W. Hagler, K. Pakbaz, A.J. Heeger, Phys. Rev. B 49 (1994) 10968.

[15] T.W. Hagler, K. Pakbaz, A.J. Heeger, Phys. Rev. B 51 (1995) 14199. 
[16] K. Pichler, D.A. Halliday, D.D.C. Bradley, P.L. Burn, R.H. Friend, A.B. Holmes, J. Phys. Condens. Matter 5 (1993) 7155.

[17] J.M. Oberski, A. Greiner, H. Bassler, Chem. Phys. Lett. 184 (1991) 391.

[18] J. Yu, W.S. Fann, F.J. Kao, D.Y. Yang, S.H. Lin, Synth. Met. 66 (1994) 143.

[19] J. Yu, J.H. Hsu, K.R. Chuang, C.I. Chao, S.A. Chen, F.J. Kao, W.S. Fann, S.H. Lin, Synth. Met. 74 (1995) 7.

[20] J. Yu, M. Hayashi, S.H. Lin, K.-K. Liang, J.H. Hsu, W.S. Fann, C.I. Chao, K.R. Chuang, S.A. Chen, Synth. Met. 82 (1996) 159.

[21] B.E. Kohler, I.D.W. Samuel, J. Chem. Phys. 103 (1995) 6248.

[22] S. Yaliraki, R.J. Silbey, J. Chem. Phys. 104 (1996) 1245.

[23] G. Rossi, R.R. Chance, R. Silbey, J. Chem. Phys. 90 (1989) 7594.

[24] P.W. Anderson, Phys. Rev. 109 (1958) 1492.

[25] M.L. Shand, R.R. Chance, M. LePostollec, M. Schott, Phys. Rev. B. 25 (1982) 4431.

[26] O. Lhost, J.L. Bredas, J. Chem. Phys. 96 (1992) 5279.

[27] F. Wudl et al. US patent 5189136.

[28] S.H. Lin, J. Chem. Phys. 44 (1966) 3759.

[29] J. Cornil, D. Beljonne, R.H. Friend, J.L. Bredas, Chem. Phys. Lett. 223 (1994) 82.

[30] J. Cornil, D. Beljonne, C.M. Heller, I.H. Campbell, B.K. Laurich, D.L. Smith, D.D.C. Bradley, K. Mullen, J.L. Bredas, Chem. Phys. Lett. 278 (1997) 139.

[31] N. Metroplis, A.W. Rosenbluth, M.N. Rosenbluth, A.H. Teller, E. Teller, J. Chem. Phys. 21 (1953) 1087.

[32] C.H. Chang, J.C. Chang, R. Chang, J.H. Hsu, W.S. Fann, K.K. Liang, M. Hayashi, J. Yu, J.C. Jiang, S.H. Lin, E.C. Chang, K.R. Chuang, S.A. Chen, Synth. Met., in press.

[33] Z. Soos, K. Schweizer, Chem. Phys. Lett. 139 (1987) 196.

[34] J.M. Leng, S. Jeglinski, X. Wei, R.E. Benner, Z.V. Vardeny, F. Kuo, S. Mazumdar, Phys. Rev. Lett. 73 (1994) 3180. 